\title{
Asymptotic Behavior of Non-oscillatory Solutions of Second order Integro- Dynamic Equations on Time Scales
}

\section{Said R Grace, Mohamed A El-Beltagy and Sarah A Deif}

Department of Engineering Mathematics, Faculty of Engineering, Cairo University, Orman, Giza 12221,Egypt

\begin{abstract}
In this paper, we investigate some new criteria on the asymptotic behavior of non-oscillatory solutions of second order integro-dynamic equations on time-scales. We also provide some numerical examples to illustrate the relevance of the obtained results.
\end{abstract}

Keywords: Asymptotic behavior; Non-oscillatory; Integro-dynamic equations; Time scales

\section{Introduction}

In this paper we are concerned with the asymptotic behavior of the non-oscillatory solutions of second order integro-dynamic equation on time-scale of the form

$$
\left(a(t) x^{\Delta}(t)\right)^{\Delta}+\int_{0}^{t} k(t, s) F(s, x(s)) \Delta s=0
$$

We take $T \subseteq R$ to be an arbitrary time-scale with $0 \in T$ and $\operatorname{Sup} T=\infty$.

Whenever, we write $t \geq s$, we mean $t \in[s, \infty) \cap T$

We assume throughout that:

i) $a, T \rightarrow R$ and $k: T \times T \rightarrow R$ are rd-continuous and $a(t)>0$ and

$k(t, s) \geq 0$ for $t \geq s$ and $\sup _{t \geq t_{0}} \int_{0}^{t_{0}} k(t, s) \Delta s:=k<\infty$;

ii) $F: T \times R \rightarrow R \quad$ is continuous and assume that there exist $f_{1}, f_{2}: T \times R \rightarrow R$ are continuous such that $F(t, x)=f_{1}(t, x)-f_{2}(t, x)$ for $t \geq 0$

(iii) there exist constants $\beta$ and $\gamma$ which are the ratios of positive odd integers and $p_{i}(t) \in C_{r d}(T,(0, \infty)), i=1,2$ such that

$$
\begin{aligned}
& f_{1}(t, x) \geq p_{1}(t) x^{\beta} \text { and } f_{2}(t, x) \leq p_{2}(t) x^{\gamma} \text { for } x>0 \text { and } t \geq 0, \\
& f_{1}(t, x) \geq p_{1}(t) x^{\beta} \text { and } f_{2}(t, x) \leq p_{2}(t) x^{\gamma} \text { for } x<0 \text { and } t \geq 0,
\end{aligned}
$$

By solutions of equation (1.1) we mean a delta- differentiable function defined on $\mathrm{T}$ that is nontrivial in a neighborhood of $\infty$. A solution $\mathrm{x}$ of equation (1.1) is said to be oscillatory if for every $t_{1} \geq 0$ $t_{0} \geq 0$ we have inf $\mathrm{t} \geq \mathrm{t}_{1} \mathrm{x}(\mathrm{t})<0<\sup \mathrm{t} \geq \mathrm{t}_{1} \mathrm{x}(\mathrm{t})$ and non-oscillatory otherwise.

With respect to dynamic equations on time-scales, it is a fairly new topic and for general basic ideas and background, we refer the reader to $[1]$.

Oscillation and non-oscillation results for integral equations of Volterra-type are scant and only a few references exist on this subject. Related studies can be found in [2-5]. To the best of our knowledge, there appear to be no such results on asymptotic behavior of nonoscillatory solutions of equations (1.1). Therefore; the main goal of this paper is to establish some new criteria for the asymptotic behavior of non-oscillatory solutions of equation (1.1) and other related equations. Also we provide some numerical examples to illustrate the obtained results when $\mathrm{T}=\mathrm{R}$.

\section{Main Results}

We shall employ the following lemma.

Lemma 4.1: If $\mathrm{X}$ and $\mathrm{Y}$ are nonnegative [6], then

$$
X^{\lambda}+(\lambda-1) Y^{\lambda}-\lambda X Y^{\lambda-1} \geq 0, \lambda>1
$$

and

$$
X^{\lambda}-(1-\lambda) Y^{\lambda}-\lambda X Y^{\lambda-1} \leq 0, \lambda<1
$$

where equality hold if and only if $\mathrm{X}=\mathrm{Y}$.

We define $R\left(t, t_{0}\right)=\int_{t_{0}}^{t} \frac{s}{a(s)} \Delta s$ for all $t_{0} \geq 0$.

Here is our first result.

Theorem 4.1: Let conditions (i)-(iii) hold with

$$
\gamma=1 \text { and } \beta>1 \text { and suppose }
$$

$$
\lim _{t \rightarrow \infty} \frac{1}{R\left(t, t_{0}\right)} \int_{t_{0}}^{t} \frac{1}{a(v)} \int_{t_{0}}^{v} \int_{t_{0}}^{u} k(u, s) p_{1}^{\frac{1}{1-\beta}}(s) p_{2}^{\frac{\beta}{\beta-1}}(s) \Delta s \Delta u \Delta v<\infty
$$

for all $t_{0} \geq 0$. If $\mathrm{x}$ is a non-oscillatory solution of equation (1.1) for $\mathrm{t}$, then

$$
x(t)=o\left(R\left(t, t_{0}\right)\right) \text { as } t \rightarrow \infty .
$$

Proof: Let $\mathrm{x}$ be a non-oscillatory solution of equation (1.1). Hence $\mathrm{x}$ is either eventually positive or $\mathrm{x}$ is eventually negative.

First assume $\mathrm{x}$ is eventually positive. Fix $t_{0} \geq 0$. Assume $x(t)>0$ for $t \geq t_{1}$ for some $t_{1} \geq t_{0}$.

Using conditions (ii) and (iii) with $\beta=1$ and $\gamma=1$ in equation (1.1) we have

$$
\left(a(t) x^{\Delta}(t)\right)^{\Delta} \leq-\int_{0}^{t_{1}} k(t, s) F(s, x(s)) \Delta s+\int_{t_{1}}^{t} k(t, s)\left[p_{2}(s) x(s)-p_{1}(s) x^{\beta}\right] \Delta s
$$

*Corresponding author: Said R Grace, Department of Engineering Mathematics, Faculty of Engineering, Cairo University, Orman, Giza 12221,Egypt, E-mail: saidgrace@yahoo.com

Received May 08, 2013; Accepted August 25, 2013; Published August 30, 2013

Citation: Grace SR, El-Beltagy MA, Deif SA (2013) Asymptotic Behavior of Nonoscillatory Solutions of Second order Integro-Dynamic Equations on Time Scales. J Appl Computat Math 2: 134. doi:10.4172/2168-9679.1000134

Copyright: $\odot 2013$ Grace SR, et al. This is an open-access article distributed under the terms of the Creative Commons Attribution License, which permits unrestricted use, distribution, and reproduction in any medium, provided the original author and source are credited. 
for all $t \geq t_{1}$. Let

$m:=\max \left\{|F(t, x(t))|: t \in\left[0, t_{1}\right] \cap T\right\}$

By assumption (i), we have $\left|-\int_{0}^{t_{1}} k(t, s) F(s, x(s)) \Delta s\right| \leq \int_{0}^{t_{1}} k(t, s)|F(s, x(s))| \Delta s$

$\leq m k \mid:=b$

Hence from (2.5) and (2.6), we get

$\left(a(t) x^{\Delta}(t)\right)^{\Delta} \leq b+\int_{t_{1}}^{t} k(t, s)\left[p_{2}(s) x(s)-p_{1}(s) x^{\beta}\right] \Delta s$

If we apply (2.1) with $\lambda=\beta, X=p_{1}^{1 / \beta} x$ and $Y=\left(\frac{1}{\beta} p_{2} p_{1}^{-1 / \beta}\right)^{1 / \beta-1}$

we obtain $p_{2}(t) x(t)-p_{2}(t) x^{\beta}(t) \leq(\beta-1) \beta^{\frac{\beta}{1-\beta}} p_{1}^{\frac{1}{1-\beta}}(t) p_{2}^{\frac{\beta}{1-\beta}}(t)$ for $t \geq 0$

Substituting (2.8) into (2.7) gives

$\left(r(t) x^{\Delta}(t)\right)^{\Delta} \leq b+(\beta-1) \beta^{\frac{\beta}{1-\beta}} \int_{t_{1}}^{t} k(t, s) p_{1}^{\frac{1}{1-\beta}}(s) p_{2}^{\frac{\beta}{1-\beta}}(s) \Delta s$.

Integrating this equality from $t_{1}$ to $t$, we have

$x^{\Delta}(t) \leq \frac{a\left(t_{1}\right) x^{\Delta}\left(t_{1}\right)}{a(t)}+\left(\frac{b\left(t-t_{1}\right)}{a(t)}\right)+\frac{1}{a(t)} \int_{t_{1}}^{t} \int_{t_{1}}^{u} k(t, s)\left[(\beta-1) \beta^{\frac{\beta}{1-\beta}} p_{2}^{\frac{\beta}{1-\beta}}(s) p_{1}^{\frac{\beta}{1-\beta}}\right] \Delta s \Delta u$.

Or,

$x^{\Delta}(t) \leq \frac{c t}{a(t)}+\frac{1}{a(t)} \int_{t_{1}}^{t} \int_{t_{1}}^{u} k(t, s)\left[(\beta-1) \beta^{\frac{\beta}{1-\beta}} p_{2}^{\frac{\beta}{1-\beta}}(s) p_{1}^{\frac{1}{1-\beta}}\right] \Delta s \Delta u$,

Where

$c=\frac{a\left(t_{1}\right)\left|x^{\Delta}\left(t_{1}\right)\right|}{t_{1}}+b$.

Integrating this equality from $t_{1}$ to $t$ we get,

$|x(t)| \leq\left|x\left(t_{1}\right)\right|+c \int_{t_{1}}^{t} \frac{s}{a(s)} \Delta s+\int_{t_{1}}^{t}\left[\frac{1}{a(v)} \int_{t_{1}}^{v} \int_{t_{1}}^{u} k(v, s)\left[(\beta-1) \beta^{\frac{\beta}{1-\beta}} p_{2}^{\frac{\beta}{1-\beta}}(s) p_{1}^{\frac{1}{1-\beta}}(s)\right] \Delta s \Delta u\right] \Delta v$.

$\leq|x(t)|+c \int_{t_{0}}^{t} \frac{s}{a(s \mid)} \Delta s+\int_{t_{0}}^{t}\left[\frac{1}{a(v)} \int_{t_{0}}^{v} \int_{t_{0}}^{u} k(v, s)\left[(\beta-1) \beta^{\frac{\beta}{1-\beta}} p_{2}^{\frac{\beta}{\beta-1}}(s) p_{1}^{\frac{1}{1-\beta}}(s)\right] \Delta s \Delta u\right] \Delta v$.

Now assume $\mathrm{x}$ is eventually negative, say $\mathrm{x}(\mathrm{t})<0$ for $\mathrm{t} \geq \mathrm{t}_{1}$ for some $\mathrm{t} \geq \mathrm{t}_{0}$.

$|x(t)| \leq\left|x\left(t_{1}\right)\right|+c \int_{t_{0}}^{t} \frac{s}{a(s)} \Delta s+\int_{t_{0}}^{t}\left[\frac{1}{a(v)} \int_{t_{0}}^{v} \int_{t_{0}}^{u} k(v, s)\left[(\beta-1) \beta^{\frac{\beta}{1-\beta}} p_{2}^{\frac{\beta}{\beta-1}}(s) p_{1}^{\frac{1}{1-\beta}}(s)\right] \Delta s \Delta u\right] \Delta v$

Dividing by both sides of this inequality $R\left(t, t_{0}\right)$ and using (2.3), we see that $\mathrm{x}$ satisfies (2.4).

This completes the proof.

Next, we give the following simple result.

Theorem 4.2: Let conditions (i) and (ii) hold with $\mathrm{f}_{2}=0$ and $x f_{1}(t, x)>0$ for $x \neq 0$ and $t \geq 0$.

If $\mathrm{x}$ is a non-oscillatory solution of equation (1.1), then (2.4) holds.

Proof: Let $\mathrm{x}(\mathrm{t})$ be a non-oscillatory solution of equation (1.1) with $\mathrm{f}_{2}=0$. First assume $\mathrm{x}$ is eventually positive Fix $t_{0} \geq 0$. Assume say $x(t)>0$ for some $t_{1} \geq t_{0}$. From equation (1.1) we find

$$
\begin{aligned}
& \left(a(t) x^{\Delta}(t)\right)^{\Delta}=-\int_{0}^{t} k(t, s) f_{1}(s, x(s)) \Delta s \\
& \leq-\int_{0}^{t} k(t, s) f_{1}(s, x(s)) \Delta s
\end{aligned}
$$

Using (2.6) in the above inequality, we obtain

$\left(a(t) x^{\Delta}(t)\right)^{\Delta} \leq b$ for $t \geq t_{1}$

The rest of the proof is similar to that of Theorem 2.1 and hence is omitted

The following corollary is immediate.

Corollary 4.1: Let conditions (i) and (ii) hold with $\mathrm{f}_{2}=0$ and

$x f_{1}(t, x)>0$ for $x \neq 0$ and $t \geq 0$. and

$\int_{t_{0}}^{\infty} \frac{s}{a(s)} \Delta s<\infty$ for any $t_{0} \geq 0$.

If $x$ is a non-oscillatory solution of equation (1.1) then $x(t)$ is bounded.

Theorem 4.3: Let conditions (i)-(iii) hold with $\beta=1$ and $\gamma=1$ and suppose

$$
\lim _{t \rightarrow \infty} \frac{1}{R\left(t, t_{0}\right)} \int_{t_{0}}^{t} \frac{1}{a(v)}\left(\int_{t_{0}}^{v} \int_{t_{0}}^{u} k(u, s) p_{1}^{\frac{\gamma}{\gamma-1}}(s) p_{2}^{\frac{1}{1-\gamma}}(s) \Delta s \Delta u\right) \Delta v<\infty
$$

for all $t_{0} \geq 0$. If $\mathrm{x}$ is a non-oscillatory solution of equation (1.1), then (2.4) holds.

Proof: Let $\mathrm{x}$ be a non-oscillatory solution of equation (1.1).First assume that $\mathrm{x}$ is eventually positive.

Fix $t_{0} \geq 0$. Assume $x(t)>0$ for $t \geq t_{1}$ for some $t_{1} \geq t_{0}$ Using condition (ii) and (iii) with $\beta=1$ and $\gamma<1$ in equation (1.1) we have

$\left(a(t) x^{\Delta}(t)\right)^{\Delta} \leq-\int_{0}^{t_{1}} k(t, s) F(s, x(s)) \Delta s+\int_{t_{1}}^{t} k(t, s)\left[p_{2}(s) x^{\gamma}(s)-p_{1}(s) x\right] \Delta s$

By applying (2.2) with $\lambda=\gamma, P={ }^{1 / \gamma} x$ and $Y=\left(\frac{1}{\gamma} p_{1} p_{2}^{\frac{-1}{\gamma}}\right)^{\frac{1}{\gamma-1}}$

We have

$p_{2}(t) x^{\gamma}(t)-p_{1}(t) x(t) \leq(1-\gamma) \gamma^{\frac{\gamma}{1-\gamma}} p_{1}^{\frac{\gamma}{\gamma-1}}(t) p_{2}^{\frac{1}{1-\gamma}}(t)$ for $t \geq 0$.

Using (2.12) in (2.11) we have

$\left(a(t) x^{\Delta}(t)\right)^{\Delta} \leq b+1(1-\gamma) \gamma^{\frac{\gamma}{1-\gamma}} \int_{t_{1}}^{t} k(t, s) p_{1}^{\frac{\gamma}{\gamma-1}}(s) p_{2}^{\frac{1}{1-\gamma}}(s) \Delta s$.

where $b$ is as in (2.6).

The rest of the proof is similar to that of Theorem 4.1 and hence is omitted.

Finally, we present the following results with different nonlinearities i.e. with $\beta>1$ and $\gamma<1$.

Theorem 4.4: Let conditions (i)-(iii) hold with $\beta>1$ and $\gamma<1$ and assume that there exists a positive rd-continuous function $\xi: T \rightarrow T$ such that

$$
\lim _{t \rightarrow \infty} \frac{1}{R\left(t, t_{0}\right)} \int_{t_{0}}^{t}\left(\frac{1}{a(v)} \int_{t_{0}}^{v} \int_{t_{0}}^{s} k(s, u)\left[c_{1} \xi^{\frac{\beta}{\beta-1}}(u)+p_{1}^{\frac{1}{1-\beta}}(u)+c_{2} \xi^{\frac{\gamma}{\gamma-1}}(u)\right] \Delta u \Delta s\right) \Delta v<\infty
$$

for all $t_{0} \geq 0$, where $c_{1}=(\beta-1) \beta^{\frac{\beta}{1-\beta}}$ and $c_{2}=(1-\gamma) \gamma^{\frac{\lambda}{1-\gamma}}$ for all $t_{0} \geq 0$ If $\mathrm{x}$ is a non-oscillatory solution of equation (1.1), then (2.4) holds.

Proof: Let $\mathrm{x}$ be a non-oscillatory solution of equation (1.1). First assume $\mathrm{x}$ is eventually positive. Fix $t_{0} \geq 0$ Assume $x(t)>0$ for $t \geq t_{1}$ for some $t_{1} \geq t_{0}$ Using conditions (ii) and (iii) in equation (1.1) we obtain 


$$
\begin{aligned}
& \left(a(t) x^{\Delta}(t)\right)^{\Delta}=-\int_{0}^{t_{1}} k(t, s) F(s, x(s)) \Delta s+\int_{t_{1}}^{t} k(t, s)\left[\xi(s) x(s)-p_{1}(s) x^{\beta}(s)\right] \Delta s \\
& +\int_{t_{1}}^{t} k(t, s)\left[p_{2}(s) x^{\gamma}(s)-\xi(s) x(s)\right] \Delta s .
\end{aligned}
$$

As in the proof of Theorems 2.1 and 2.3, we can easily find

$$
\left(a(t) x^{\Delta}(t)\right)^{\Delta} \leq b+\int_{t_{0}}^{t} k(t, s)\left[(\beta-1) \beta^{\frac{\beta}{1-\beta}} \xi \beta^{\frac{\beta}{\beta-1}}(s) p_{1}^{\frac{1}{1-\beta}}(s)+(1-\gamma) \gamma^{\frac{\gamma}{1-\gamma}} \xi^{\frac{\gamma}{1-\gamma}}(s) p_{2}^{\frac{1}{1-\gamma}}(s)\right] \Delta s
$$

The rest of the proof is similar to that of Theorem 4.1 and hence is omitted. Theorem 4.4 can be re-stated as follows:

Theorem 4.5: Let conditions (i)-(iii) hold with $\beta>1$ and $\gamma<1$ and assume that there exists a positive rd-continuous function $\xi: T \rightarrow T$ such that

$$
\lim _{t \rightarrow \infty} \frac{1}{R\left(t, t_{0}\right)} \int_{t_{0}}^{t}\left(\frac{1}{a(v)} \int_{t_{0}}^{v} \int_{t_{0}}^{s} k(s, u)\left[\xi^{\frac{\beta}{\beta-1}}(u) p_{1}^{\frac{1}{1-\beta}}(u)\right] \Delta u \Delta s\right) \Delta v<\infty
$$

and

$$
\lim _{t \rightarrow \infty} \frac{1}{R\left(t, t_{0}\right)} \int_{t_{0}}^{t}\left(\frac{1}{a(v)} \int_{t_{0}}^{v} \int_{t_{0}}^{s} k(s, u)\left[\xi^{\frac{\gamma}{\gamma-1}}(u) p_{2}^{\frac{1}{1-\gamma}}(u)\right] \Delta u \Delta s\right) \Delta v<\infty
$$

for all $t_{0} \geq 0$. If $\mathrm{x}$ is a non-oscillatory solution of equation (1.1), then (2.4) holds.

For the case of forced integro-differential equation

$$
\left(r(t)\left(x^{\Delta}(t)\right)\right)^{\Delta}+\int_{0}^{t} a(t, s) F(s, x(s)) \Delta s=e(t)
$$

Where $e: T \rightarrow R$. Now, if in addition to the hypotheses of all the results presented above, we assume that is rd-contiuous function.

$$
\lim _{t \rightarrow \infty} \frac{1}{R\left(t, t_{0}\right)} \int_{t_{0}}^{t}\left(\frac{1}{r(v)} \int_{t_{0}}^{v}|e(s)| \Delta \mid s\right) \Delta v<\infty .
$$

then the conclusion of these results hold for equation (2.15).

\section{Numerical Examples}

As we already mentioned that the results of the present paper are new even for the cases when $T=R$ i.e., the continuous case or when $\mathrm{T}=\mathrm{Z}$ i.e., the discrete case.

As a numerical illustration of Theorems 4.3, 4.4 and 4.1 respectively, let us consider the following equation

$$
\left.\left(t x^{\prime}(t)\right)\right)^{\prime}+\int_{t_{0}}^{t} \frac{t}{\left(t^{2}+s^{2}\right)}\left(x^{\gamma}(s)-x^{\beta}(s)\right) d s=0 ; t_{0} \geq 0
$$

with initial conditions $x\left(t_{0}\right)=x_{0}$ and $x^{\prime}\left(t_{0}\right)=x_{0}{ }^{\prime}$. Equation (3.1) can be converted to two simultaneous first order ordinary differential equations by substituting $t x^{\prime}=y$. This will lead to the following system:

$$
\begin{aligned}
& x^{\prime}(t)=\frac{y(t)}{t} ; x\left(t_{0}\right)=x_{0} \\
& y^{\prime}(t)=-\int_{t_{0}}^{t}-\int_{t_{0}}^{t} \frac{1}{\left(t^{2}-s^{2}\right)}\left(x^{\gamma}(s)-x^{\beta}(s)\right) d s ; y\left(t_{0}\right)=t_{0} x_{0}^{\prime}
\end{aligned}
$$

Many numerical techniques can be used to solve (3.2). In the current work, the second order, accurate modified Euler technique is considered. The time interval $\left[\mathrm{t}_{0}, \mathrm{~T}\right]$ will be divided into $\mathrm{N}$ equal subdivisions with width for each one. The prediction and correction steps of the modified Euler technique will be:

$$
\left\{\begin{array}{c}
\bar{x}_{i+1}=x_{i}+\Delta t f\left(t_{i}, x_{i}, y_{i}\right) \\
\bar{y}_{i+1}=x_{i}+\Delta \operatorname{tg}\left(t_{i}, x_{i}, y_{i}\right) \\
x_{i+1}=x_{i}+0.5 \Delta t\left[f\left(t_{i}, x_{i}, y_{i}\right)+f\left(t_{i+1}, \bar{x}_{i+1}, \bar{y}_{i+1}\right)\right] \\
y_{i+1}=y_{i}+0.5 \Delta t\left[g\left(t_{i}, x_{i}, y_{i}\right)+g\left(t_{i+1}, \bar{x}_{i+1}, \bar{y}_{i+1}\right)\right]
\end{array}\right.
$$

Where

$$
f(t, x, y)=\frac{y}{t}
$$

and

$$
g(t, x, y)=-\int_{t_{0}}^{t} \frac{t}{\left(t^{2}-s^{2}\right)}\left(x^{\gamma}(s)-x^{\beta}(s)\right) d s
$$

The integral (3.5) can be approximated numerically at each time instant $\mathrm{t}_{\mathrm{i}}$ using the trapezoidal rule which has accuracy of $O\left(\Delta t_{i}\right)^{2}$ where $\Delta t_{i}$ is the subdivision width when dividing the interval $\left[t_{0}, t_{i}\right]$ into Ni subdivisions.

Let $t_{0}=1, T=100, x\left(t_{0}\right)=1$ and $x^{\prime}\left(t_{0}\right)$ in equation (4.3) for different values for $\gamma$ and $\beta$. Figure 1 shows the asymptotic behavior of $\mathrm{x}(\mathrm{t})$ with the time for $\gamma=1 / 3$ and $\beta=1$ [7]. The solution $\mathrm{x}(\mathrm{t})$ asymptotes to a straight line of slope 3.73 approximately after $t=70$. Increasing the value of $\beta$ to be equal 3, the line slope will increase to 74.5 approximately after $t=90$, as shown in Figure 2. Increasing $\gamma$ to be equal 1 with $\beta=3$, will have negligible effect on the solution behavior, as shown in Figure 3. In this case the solution asymptotes, approximately, to the same straight line in Figure 2.

\section{General Remarks}

We conclude by presenting several remarks and extensions of the results given above

i) The results presented in this paper are new for $T=R$ and $T=Z$.

ii) The results of this paper are presented in a form which is essentially new for equation (1.1) with different nonlinearities. Corollaries similar to Corollary 2.1 can be obtained. Here we omit the details.

iii) The results of this paper will remain the same if we replace (1.2) of assumption (i) by

$$
\sup _{0 \leq s \leq t_{0}} k(t, s)=k *<\infty
$$

with $k=k * t_{0}$ and $t_{0}>0$,

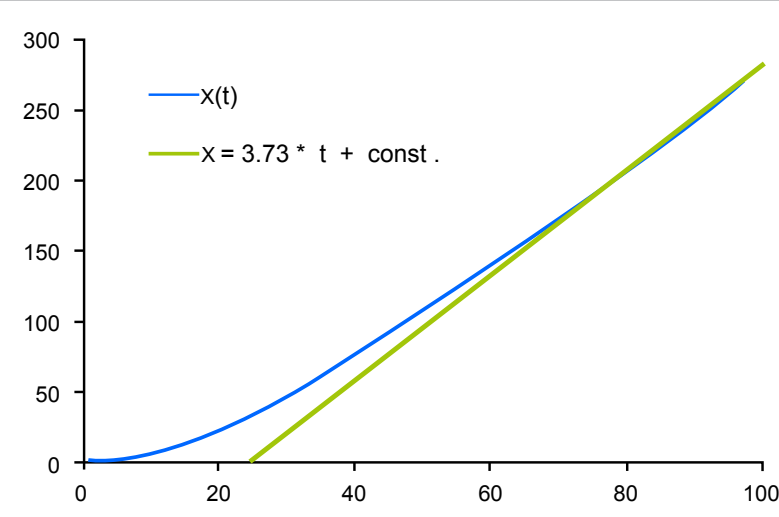

Figure 1: The solution $\mathrm{x}(\mathrm{t})$ and the approximate asymptotic line for $\gamma=1 / 3$ and $\beta=1$. 


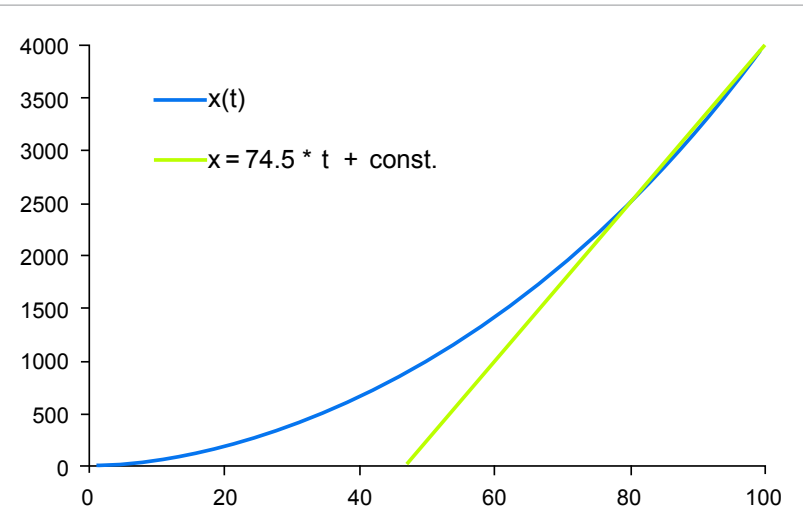

Figure 2: The solution $\mathrm{x}(\mathrm{t})$ and the approximate asymptotic line for $\gamma=1 / 3$ and $\beta=3$.

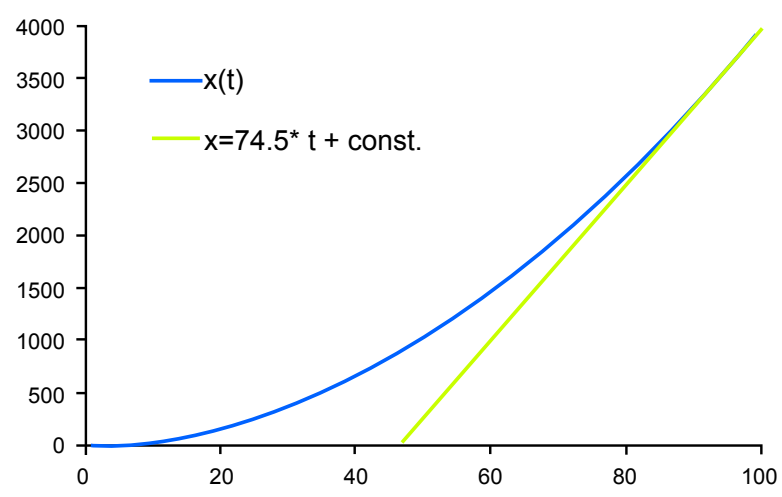

Figure 3: The solution $\mathrm{x}(\mathrm{t})$ and the approximate asymptotic line for $\gamma=1$ and $\beta=3$.

or, we replace condition (1.2) of assumption (i) by:

there exist rd-continuous functions $k, m: T \rightarrow R^{+}$such that $a(t, s) \leq k(t) m(s)$ for all $t \geq s$

$$
\begin{aligned}
& \sup _{t \geq 0} k(t)=: k_{1}<\infty \\
& \text { and } \sup _{t \geq 0} \int_{0}^{t} m(s) \Delta s=k_{2}<\infty .
\end{aligned}
$$

with $\mathrm{k}_{\mathrm{n}} \mathrm{k}_{1} \mathrm{k}_{2}$. The details are left to the reader.

iv) The technique offered in this paper can be employed to Volterra integral equations on timescales of the form

$$
x(t)+\int_{0}^{t} k(t, s) F(s, x(s)) \Delta s=0 .
$$

As example, we reformulate Theorem 2.1 and find

Theorem 4.1 Let conditions (ii) and (iii) hold with $\beta=1$ and $\gamma=1$ and suppose

$$
\lim _{t \rightarrow \infty} \int_{t_{0}}^{t} k(t, s) p_{2}^{\frac{\beta}{1-\beta}}(s) p_{1}^{\frac{1}{1-\beta}}(s) \Delta s<\infty,
$$

for all $t_{0} \geq 0$. If $\mathrm{x}$ is a non-oscillatory solution of equation (4.3), then $\mathrm{x}$ is bounded Similar results can be obtained and the details are left to the reader.

In addition to the hypotheses of Theorems 2.1-2.6, assume

$$
\lim _{t \rightarrow \infty} R\left(t, t_{0}\right)<\infty \text {. }
$$

If $\mathrm{x}$ is a non-oscillatory solution of equation (1.1), then $\mathrm{x}$ is bounded

v) The results of this paper can be extended easily to delay integrodynamic equations of the form.

$$
\left(a(t) x^{\Delta}(t)\right)^{\Delta}+\int_{0}^{t} k(t, s) F(s, x(g(s))) \Delta s=0
$$

Where $g: T \rightarrow T$ is rd-continuous function, $g(t) \leq t, g^{\Delta}(t) \geq 0$ for $\lim _{t \rightarrow \infty} g(t)=\infty$. The formulation of the results is left to the reader.

We note that we can reformulate the obtained results for the time scales $\mathrm{T}=\mathrm{R}$ (the continuous case), $\mathrm{T}=\mathrm{Z}$ (the discrete case), $T=N_{0}^{2}, T=h \mathrm{Z}$ with $h>0$...etc. see [1]. The details are left to the reader.

\section{References}

1. Bohner M, Peterson A (2001) Dynamic Equations on Time-Scales: An Introduction with Applications, Boston, Birkhauser.

2. Karakostas G, Stavroulakis IP, Wu Y (1993) Oscillation of Volterra integral equations. Tohoku Math J 45: 583-605.

3. Onose H (1990) On Oscillation of Volterra integral equations and first order functional differential equations. Hiroshima Math J 20: 223-229.

4. Parhi N, Misra N (1983) On oscillatory and non-oscillatory behavior of solutions of Volterra integral equations. J Math Anal Appl 94: 137-149.

5. Singh B (1995) On the oscillation of Volterra integral equation. Czech Math J 45: 699-707.

6. Hardy GH, Littlewood IE, Polya G (1959) Inequalities, University Press, Cambridge.

7. Bohner M, Stvic S (2007) Asymptotic behavior of second order dynamic equations. Applied Math Comput 188: 1503-1512. 